\title{
On relations for the multiple $q$-zeta values
}

\author{
OKUDA, Jun-ichi
}

\author{
TAKEYAMA, Yoshihiro
}

\begin{abstract}
We prove a new relation for the multiple $q$-zeta values $\left(\mathrm{M} q Z \mathrm{~V}^{\prime} \mathrm{s}\right)$. It is a $q$-analogue of the Ohno-Zagier relation for the multiple zeta values (MZV's). We discuss the problem to determine the dimension of the space spanned by $\mathrm{M} q Z V^{\prime}$ s over $\mathbb{Q}$, and present an application to MZV.
\end{abstract}

\section{Introduction}

In this paper we prove a new relation for a certain class of $q$-series called multiple $q$-zeta values (MqZV's, for short).

Let us recall the definition of MqZV [1,9]. We call a sequence of ordered positive integers $\mathbf{k}=\left(k_{1}, \ldots, k_{r}\right)$ an index. The weight, depth and height of the index are defined by $|\mathbf{k}|:=k_{1}+\cdots+k_{r}$, dep $\mathbf{k}:=r$ and ht $\mathbf{k}:=\#\left\{j \mid k_{j} \geq 2\right\}$, respectively. The index is said to be admissible if and only if $k_{1} \geq 2$.

Definition 1. For $0<q<1$ and an admissible index $\mathbf{k}$, the multiple q-zeta value (MqZV) is defined by

$$
\zeta[\mathbf{k}]:=\sum_{n_{1}>n_{2}>\cdots>n_{r}>0} \frac{q^{n_{1}\left(k_{1}-1\right)+n_{2}\left(k_{2}-1\right)+\cdots+n_{r}\left(k_{r}-1\right)}}{\left[n_{1}\right]^{k_{1}\left[n_{2}\right]^{k_{2}} \cdots\left[n_{r}\right]^{k_{r}}}}
$$

where $[n]$ is the q-integer

$$
[n]:=\frac{1-q^{n}}{1-q} .
$$

Note that $0<1 /[n] \leq 1$ for any positive integer $n$. Hence the right hand side of (1) is absolutely convergent if $k_{1}>1$. In particular it is well defined as a $q$-series if $\mathbf{k}$ is admissible.

By taking the limit $q \rightarrow 1-$ of $\mathrm{M} q \mathrm{ZV}$, we obtain the multiple zeta value (MZV, for short):

$$
\lim _{q \rightarrow 1-} \zeta[\mathbf{k}]=\zeta(\mathbf{k}):=\sum_{n_{1}>n_{2}>\cdots>n_{r}>0} \frac{1}{n_{1}^{k_{1}} n_{2}^{k_{2}} \cdots n_{r}^{k_{r}}} .
$$

For MZV's there are many linear relations and algebraic ones over $\mathbb{Q}$. The examples are the cyclic sum formula [3], the Ohno relation [5] and the Ohno-Zagier relation 
[6]. These relations do not suffice to give all relations of MZV's, and as a time the proof is much technical. However these relations have quite beautiful structures and it is valuable to construct explicit relations.

In [1], Bradley proved the q-analogue of the cyclic sum formula and the Ohno relations. They are linear relations over $\mathbb{Q}$ among $M q Z V^{\prime}$ s of fixed weight. Moreover it is shown that MqZV's of type $\zeta[m+2,1, \ldots, 1]$ are given by a polynomial of $\zeta[2], \zeta[3], \ldots$ with rational coefficients (see Theorem 15 of [1]).

In the present paper, we give a $q$-analogue of the Ohno-Zagier relation, which is a generalization of Bradley's result:

Theorem 1. We define a generating function of MqZV's as follows:

$$
\Phi_{0}[x, y, z]:=\sum_{k, r, s=0}^{\infty}\left\{\sum_{\substack{\mathbf{k} \\|\mathbf{k}|=k, \operatorname{dep} \mathbf{k}=r, \text { ht } \mathbf{k}=s}} \zeta[\mathbf{k}]\right\} x^{k-r-s} y^{r-s} z^{s-1}
$$

Then

$$
\begin{aligned}
& 1+(z-x y) \Phi_{0} \\
& =\exp \left(\sum_{n=2}^{\infty} \zeta[n] \sum_{m=0}^{\infty} \frac{(q-1)^{m}}{m+n}\left(x^{m+n}+y^{m+n}-\left(\alpha^{m+n}+\beta^{m+n}\right)\right)\right) .
\end{aligned}
$$

Here $\alpha^{m+n}+\beta^{m+n}$ is a polynomial in $x, y$ and $z$ determined by

$$
\alpha+\beta=x+y+(q-1)(z-x y) \text { and } \alpha \beta=z .
$$

By taking the limit $q \rightarrow 1-$, we obtain the Ohno-Zagier relation for MZV's. The Ohno-Zagier relation for MZV's explains when the combinations of MZV's are in the algebra generated by Riemann's zeta values $\zeta(k)$ over $\mathbb{Q}$. Unfortunately the product of MqZV's is not closed in Q-vector space spanned by MqZV's, however it is closed in $\mathbb{Q}[(1-q)]$-module and preserves the weights by counting the weight of $(1-q)$ by $1[1,9]$. Now we define the modified MqZV $\bar{\zeta}[\mathbf{k}]$ by

$$
\bar{\zeta}[\mathbf{k}]:=(1-q)^{-|\mathbf{k}|} \zeta[\mathbf{k}] .
$$

Then the product of modified MqZV's is closed in the Q-vector space spanned by them. The relation (3) is rewritten as follows:

$$
1+(z-x y) \bar{\Phi}_{0}=\exp \left(\sum_{n=2}^{\infty} \bar{\zeta}[n] \sum_{m=0}^{\infty} \frac{(-1)^{m}}{m+n}\left(x^{m+n}+y^{m+n}-\left(\bar{\alpha}^{m+n}+\bar{\beta}^{m+n}\right)\right)\right) .
$$

Here $\bar{\Phi}_{0}$ is defined by (2) with $\zeta[\mathbf{k}]$ replaced by $\bar{\zeta}[\mathbf{k}]$, and

$$
\bar{\alpha}+\bar{\beta}=x+y-(z-x y) \text { and } \bar{\alpha} \bar{\beta}=z .
$$


Thus the relation (3) explains when the linear combinations of the modified MqZV's are in the algebra generated by $\bar{\zeta}[n](n \geq 2)$ over $\mathbb{Q}$. Moreover, by using the $q$-stuffle rule $[1,9]$

$$
\begin{aligned}
\bar{\zeta}[k] \bar{\zeta}\left[k_{1}, \ldots, k_{r}\right] & =\sum_{i=0}^{r} \bar{\zeta}\left[k_{1}, \ldots, k_{i}, k, k_{i+1}, \ldots, k_{r}\right] \\
& +\sum_{i=1}^{r} \bar{\zeta}\left[k_{1}, \ldots, k_{i}+k, \ldots, k_{r}\right] \\
& +\sum_{i=1}^{r} \bar{\zeta}\left[k_{1}, \ldots, k_{i}+k-1, \ldots, k_{r}\right],
\end{aligned}
$$

we can obtain some linear relations for modified MqZV's from (5).

The rest of the paper is organized as follows. In section 2 we prove Theorem 1 . In section 3 we discuss the problem to determine dimensions of certain spaces spanned by modified MqZV's over $Q$. In the study of MZV it is an important problem to determine the dimension of the $Q$-vector space spanned by MZV's of a fixed weight (see, e.g. [8]). We consider a similar problem in the case of $\mathrm{M} q \mathrm{ZV}$ and present some observations.

\section{Proof of Theorem 1}

In the proof of Theorem 1, we make use of the multiple $q$-polylogarithms $[1,9]$ :

$$
\operatorname{Li}_{k_{1}, \ldots, k_{r}}\left[t_{1}, \ldots, t_{r}\right]:=\sum_{n_{1}>\cdots>n_{r}>0} \frac{t_{1}^{n_{1}} \cdots t_{r}^{n_{r}}}{\left[n_{1}\right]^{k_{1}} \cdots\left[n_{r}\right]^{k_{r}}} .
$$

Since in the following only needed is the above function with $t_{2}=\cdots=t_{r}=1$, we use the abbreviation

$$
\operatorname{Li}_{\mathbf{k}}[t]:=\operatorname{Li}_{\mathbf{k}}[t, 1, \ldots, 1]
$$

Then the function $\mathrm{Li}_{\mathbf{k}}[t]$ is related to $\mathrm{M} q \mathrm{ZV}$ as follows:

$$
\begin{aligned}
\operatorname{Li}_{k_{1}, \ldots, k_{r}}[q]=\sum_{a_{1}=2}^{k_{1}} \sum_{a_{2}=1}^{k_{2}} & \cdots \sum_{a_{r}=1}^{k_{r}}\left(\begin{array}{l}
k_{1}-2 \\
a_{1}-2
\end{array}\right)\left\{\prod_{j=2}^{r}\left(\begin{array}{c}
k_{j}-1 \\
a_{j}-1
\end{array}\right)\right\} \\
& \times(1-q)^{\sum_{j=1}^{r}\left(k_{j}-a_{j}\right)} \zeta\left[a_{1}, \ldots, a_{r}\right] .
\end{aligned}
$$

Here $\left(k_{1}, \ldots, k_{r}\right)$ is an admissible index.

Now let us prove Theorem 1 . Denote by $I[k, r, s]$ the set of indices of weight $k$, depth $r$ and height $s$, and by $I_{0}[k, r, s]$ the subset consisting of admissible ones. Set

$$
G[k, r, s ; t]:=\sum_{\mathbf{k} \in I[k, r, s]} \operatorname{Li}_{\mathbf{k}}[t], \quad G_{0}[k, r, s ; t]:=\sum_{\mathbf{k} \in I_{0}[k, r, s]} \operatorname{Li}_{\mathbf{k}}[t] .
$$


By definition we set $G[0,0,0 ; t]=1$, and $G[k, r, s ; t]=0$ unless $k \geq r+s$ and $r \geq s \geq 0$. Consider the following generating functions

$$
\begin{aligned}
\Phi & :=\sum_{k, r, s \geq 0} G[k, r, s ; t] u^{k-r-s} v^{r-s} w^{s}, \\
\Phi_{0} & :=\sum_{k, r, s \geq 0} G_{0}[k, r, s ; t] u^{k-r-s} v^{r-s} w^{s-1} .
\end{aligned}
$$

The function $\Phi_{0}$ is related to $\mathrm{M} q \mathrm{ZV}$ as follows:

\section{Lemma 1.}

$$
\left.\Phi_{0}\right|_{t=q}=\frac{1}{1-(1-q) u} \sum_{k, r, s \geq 0}\left(\sum_{\mathbf{k} \in I_{0}[k, r, s]} \zeta[\mathbf{k}]\right) x^{k-r-s} y^{r-s} z^{s-1},
$$

where $x, y$ and $z$ are given by

$$
x=\frac{u}{1-(1-q) u}, \quad y=\frac{v+(1-q)(w-u v)}{1-(1-q) u}, \quad z=\frac{w}{(1-(1-q) u)^{2}} .
$$

It is easy to prove this lemma from (7).

To prove Theorem 1 we derive a $q$-difference equation satisfied by $\Phi_{0}=\Phi_{0}[t]$. Denote by $\mathcal{D}_{q}$ the $q$-difference operator

$$
\left(\mathcal{D}_{q} f\right)[t]:=\frac{f[t]-f[q t]}{(1-q) t}
$$

Then we have

$$
\mathcal{D}_{q} \operatorname{Li}_{k_{1}, \ldots, k_{r}}[t]= \begin{cases}\frac{1}{t} \operatorname{Li}_{k_{1}-1, k_{2}, \ldots, k_{r}}[t], & k_{1} \geq 2 \\ \frac{1}{1-t} \operatorname{Li}_{k_{2}, \ldots, k_{r}}[t], & k_{1}=1 .\end{cases}
$$

These relations can be rewritten in terms of $G[k, r, s ; t]$ and $G_{0}[k, r, s ; t]$ as follows:

$$
\begin{aligned}
& \mathcal{D}_{q} G_{0}[k, r, s ; t] \\
& \quad=\frac{1}{t}\left(G[k-1, r, s-1 ; t]-G_{0}[k-1, r, s-1 ; t]+G_{0}[k-1, r, s ; t]\right), \\
& \mathcal{D}_{q}\left(G[k, r, s ; t]-G_{0}[k, r, s ; t]\right)=\frac{1}{1-t} G[k-1, r-1, s ; t]
\end{aligned}
$$

or, in terms of generating functions,

$$
\mathcal{D}_{q} \Phi=\frac{1}{v t}\left(\Phi-1-w \Phi_{0}\right)+\frac{u}{t} \Phi_{0}, \quad \mathcal{D}_{q}\left(\Phi-w \Phi_{0}\right)=\frac{v}{1-t} \Phi
$$


Eliminate $\Phi$ using the formula

$$
\mathcal{D}_{q}(t f[t])=q t \cdot \mathcal{D}_{q} f[t]+f[t]
$$

Then we obtain

$$
q t(1-t) \mathcal{D}_{q}^{2} \Phi_{0}+((1-u)(1-t)-v t) \mathcal{D}_{q} \Phi_{0}+(u v-w) \Phi_{0}=1
$$

This is an equation for the power series $\Phi_{0}=\Phi_{0}[t]$. Note that $\Phi_{0}(0)=0$. There is a unique solution to (10) vanishing at $t=0$. To write down the solution we introduce the $q$-hypergeometric function

$$
\phi(a, b, c ; t):=\sum_{n=0}^{\infty} t^{n} \prod_{j=1}^{n} \frac{\left(1-a q^{j-1}\right)\left(1-b q^{j-1}\right)}{\left(1-q^{j}\right)\left(1-c q^{j-1}\right)}
$$

Then the solution is given by

$$
\Phi_{0}[u, v, w ; t]=\frac{1}{u v-w}\left(1-\phi\left(a, b, c ; \frac{c t}{q a b}\right)\right) .
$$

Here $a, b$ and $c$ are defined in terms of $u, v$ and $w$ as follows:

$$
a=\frac{1}{1-(1-q)\left(u-\alpha_{0}\right)}, \quad b=\frac{1}{1-(1-q)\left(u-\beta_{0}\right)}, \quad c=\frac{q}{1-(1-q) u},
$$

where $\alpha_{0}$ and $\beta_{0}$ are determined by

$$
\alpha_{0}+\beta_{0}=u+v, \quad \alpha_{0} \beta_{0}=w
$$

Now we use Heine's q-analogue of Gauss' summation formula (see, e.g. [2]):

$$
\phi\left(a, b, c ; \frac{c}{a b}\right)=\prod_{n=0}^{\infty} \frac{\left(1-a^{-1} c q^{n}\right)\left(1-b^{-1} c q^{n}\right)}{\left(1-c q^{n}\right)\left(1-a^{-1} b^{-1} c q^{n}\right)} .
$$

In our case, substituting (12), we have

$$
\phi\left(a, b, c ; \frac{c}{a b}\right)=\prod_{n=1}^{\infty} \frac{\left(1-\frac{q^{n}}{[n]} \alpha\right)\left(1-\frac{q^{n}}{[n]} \beta\right)}{\left(1-\frac{q^{n}}{[n]} x\right)\left(1-\frac{q^{n}}{[n]} y\right)} .
$$

Here $x$ and $y$ are given by (8), and $\alpha$ and $\beta$ are defined by

$$
\alpha=\frac{\alpha_{0}}{1-(1-q) u}, \quad \beta=\frac{\beta_{0}}{1-(1-q) u},
$$


or equivalently determined by

$$
\alpha+\beta=x+y+(q-1)(z-x y), \quad \alpha \beta=z .
$$

From Lemma 1, (11), (13) and the formula

$$
\begin{aligned}
\log \prod_{n=1}^{\infty}\left(1-\frac{q^{n}}{[n]} s\right)=\frac{1}{q-1} & \log (1-s(q-1)) \sum_{n=1}^{\infty} \frac{q^{n}}{[n]} \\
& -\sum_{n=2}^{\infty} \zeta[n] \sum_{m=0}^{\infty} \frac{(q-1)^{m}}{m+n} s^{m+n},
\end{aligned}
$$

we obtain Theorem 1 .

\section{Discussion}

Now we consider the space spanned by the modified MqZV's (4). We regard the modified $\mathrm{M} q \mathrm{ZV}$ as a formal power series of $q$, and define subspaces of $\mathbb{Q}[[q]]$ by

$$
Z_{k}:=\sum_{|\mathbf{k}|=k} \mathbb{Q} \bar{\zeta}[\mathbf{k}], \quad Z_{\leq k}:=\sum_{2 \leq|\mathbf{k}| \leq k} \mathbb{Q} \bar{\zeta}[\mathbf{k}]
$$

Let us consider the problem to determine the dimension of the spaces over $\mathbb{Q}$. In principle, a lower bound for the dimension can be obtained as follows. Expand $\operatorname{MqZV} \bar{\zeta}[\mathbf{k}]$ as a power series of $q$ :

$$
\bar{\zeta}[\mathbf{k}]=\sum_{n=0}^{\infty} a_{n}(\mathbf{k}) q^{n}, \quad a_{n}(\mathbf{k}) \in \mathbb{Z}_{\geq 0} .
$$

Note that $a_{n}(\mathbf{k})=0$ if $n<|\mathbf{k}|-1$. Recall that the number of admissible indices of weight $k$ is equal to $2^{k-2}$. Now consider the following square matrices

$$
A_{k}:=\left(a_{n}(\mathbf{k})\right)_{(\mathbf{k}, n) \in I_{k^{\prime}}} \quad A_{\leq k}:=\left(a_{n}(\mathbf{k})\right)_{(\mathbf{k}, n) \in I_{\leq k}}
$$

where $I_{k}$ and $I_{\leq k}$ are sets of indices defined by

$$
\begin{aligned}
I_{k} & :=\left\{(\mathbf{k}, n)|| \mathbf{k} \mid=k, k-1 \leq n \leq k+2^{k-2}-2\right\} \\
I_{\leq k} & :=\left\{(\mathbf{k}, n)|2 \leq| \mathbf{k} \mid \leq k, 1 \leq n \leq k+2^{k-2}-2\right\} .
\end{aligned}
$$

Then we have

$$
\operatorname{rank} A_{k} \leq \operatorname{dim} Z_{k}, \quad \operatorname{rank} A_{\leq k} \leq \operatorname{dim} Z_{\leq k}
$$

Let $d_{k}$ be the conjectured dimension of the space of MZV's of weight $k$ over $\mathbb{Q}$ in [8]. It is shown that $d_{k}$ gives the upper bound of the dimension of MZV's [7] .

The values of rank $A_{k}$ and $\operatorname{rank} A_{\leq k}$ for $2 \leq k \leq 10$ are listed in Table 1. In [1], the 
Table. 1 The ranks of $A_{k}$ and $A_{\leq k}$.

\begin{tabular}{|c||r|r|r|r|r|r|r|r|r|}
\hline weight & 2 & 3 & 4 & 5 & 6 & 7 & 8 & 9 & 10 \\
\hline$d_{k}$ & 1 & 1 & 1 & 2 & 2 & 3 & 4 & 5 & 7 \\
\hline $\operatorname{rank} A_{k}$ & 1 & 1 & 2 & 3 & 6 & 9 & 18 & 29 & 54 \\
\hline By cyclic and Ohno & 1 & 1 & 2 & 3 & 6 & 9 & 18 & 30 & 56 \\
\hline$\sum_{j \leq k} d_{j}$ & 1 & 2 & 3 & 5 & 7 & 10 & 14 & 19 & 26 \\
\hline $\operatorname{rank} A_{\leq k}$ & 1 & 2 & 4 & 7 & 11 & 18 & 27 & 42 & 63 \\
\hline$\sum_{j \leq k} \operatorname{rank} A_{j}$ & 1 & 2 & 4 & 7 & 13 & 22 & 40 & 69 & 123 \\
\hline
\end{tabular}

Table. 2 The coefficients of $\bar{\zeta}[\mathbf{k}]$ 's up to degree 13 .

\begin{tabular}{|c||rrrrrrrrrrrrr|}
\hline $\mathbf{k}$ & $q^{1}$ & $q^{2}$ & $q^{3}$ & $q^{4}$ & $q^{5}$ & $q^{6}$ & $q^{7}$ & $q^{8}$ & $q^{9}$ & $q^{10}$ & $q^{11}$ & $q^{12}$ & $q^{13}$ \\
\hline$(2)$ & 1 & 3 & 4 & 7 & 6 & 12 & 8 & 15 & 13 & 18 & 12 & 28 & 14 \\
\hline$(3)$ & 0 & 1 & 3 & 7 & 10 & 19 & 21 & 35 & 39 & 56 & 55 & 91 & 78 \\
\hline$(4)$ & 0 & 0 & 1 & 4 & 10 & 21 & 35 & 60 & 85 & 130 & 165 & 245 & 286 \\
$(3,1)$ & 0 & 0 & 0 & 1 & 1 & 6 & 5 & 15 & 18 & 31 & 30 & 70 & 55 \\
\hline$(5)$ & 0 & 0 & 0 & 1 & 5 & 15 & 35 & 71 & 126 & 215 & 330 & 511 & 715 \\
$(4,1)$ & 0 & 0 & 0 & 0 & 0 & 1 & 1 & 5 & 7 & 16 & 17 & 47 & 42 \\
$(3,2)$ & 0 & 0 & 0 & 0 & 1 & 2 & 7 & 13 & 24 & 42 & 69 & 97 & 149 \\
\hline$(6)$ & 0 & 0 & 0 & 0 & 1 & 6 & 21 & 56 & 126 & 253 & 462 & 798 & 1287 \\
$(5,1)$ & 0 & 0 & 0 & 0 & 0 & 0 & 0 & 1 & 1 & 6 & 6 & 23 & 22 \\
$(4,2)$ & 0 & 0 & 0 & 0 & 0 & 0 & 1 & 2 & 7 & 13 & 30 & 45 & 88 \\
$(3,3)$ & 0 & 0 & 0 & 0 & 0 & 1 & 3 & 10 & 22 & 47 & 85 & 154 & 244 \\
$(4,1,1)$ & 0 & 0 & 0 & 0 & 0 & 0 & 0 & 0 & 1 & 1 & 2 & 9 & 9 \\
$(3,2,1)$ & 0 & 0 & 0 & 0 & 0 & 0 & 0 & 1 & 1 & 4 & 9 & 14 & 23 \\
\hline
\end{tabular}

cyclic sum formula and the Ohno relation are proved for MqZV's. These relations have the same form as the corresponding ones for MZV's and also have the same contributions to the dimension of MqZV's. The fourth row in Table 1 shows the upper bound for the dimension of $Z_{k}$ which follows from the cyclic sum formula and the Ohno relation [4].

We show some data of $a_{n}(\mathbf{k})$ for $n \leq 13$ and $|\mathbf{k}| \leq 6$ in Table 2. Any modified $\mathrm{M} q \mathrm{ZV}$ of weight less than 7 is given by a linear combination of ones in the list above from the cyclic sum formula and the Ohno relation.

Now we discuss two points. First, at weight 9 there is a gap between rank $A_{9}$ and the upper bound in the fourth row. This shows a possibility that the cyclic sum formula and the Ohno relation are not sufficient to determine the dimension of $Z_{k}$. 
In fact, the following equality holds up to $q^{269}$ :

$$
\begin{array}{rr}
4 \zeta[7,2]+6 \zeta[6,3]-\zeta[5,4]-\zeta[4,5]-6 \zeta[6,2,1]-6 \zeta[6,1,2] & \\
-2 \zeta[5,3,1]-7 \zeta[5,2,2]-3 \zeta[5,1,3]+2 \zeta[4,4,1]-\zeta[4,3,2] & \\
+\zeta[3,5,1]+\zeta[3,2,4]-3 \zeta[2,5,2]+2 \zeta[5,2,1,1] & \\
+2 \zeta[5,1,2,1]+2 \zeta[5,1,1,2]+\zeta[3,3,1,2]-\zeta[3,2,3,1] & \\
-4 \zeta[3,2,2,2]-\zeta[3,2,1,3]-2 \zeta[2,2,3,2]+\zeta[2,1,3,3] \stackrel{?}{=} 0
\end{array}
$$

It is linearly independent of the cyclic sum formula and the Ohno relation. The relation (14) is correct in the limit as $q \rightarrow 1-[4]$.

Next, if the weight is greater than 5 , the equality $\operatorname{rank} A_{\leq k}=\sum_{j=2}^{k} \operatorname{rank} A_{j}$ breaks. This shows that there may exist linear relations among the modified MqZV's of different weights. For example, as observed in the data above, the following relations seem to hold:

$$
\begin{aligned}
-\bar{\zeta}[3,1]+ & \bar{\zeta}[5]-3 \bar{\zeta}[4,1]-3 \bar{\zeta}[3,2] \\
& -\bar{\zeta}[6]-3 \bar{\zeta}[4,2]+6 \bar{\zeta}[3,3] \stackrel{?}{=} 0 \\
-2 \bar{\zeta}[3,1]+ & 2 \bar{\zeta}[5]-6 \bar{\zeta}[4,1]-9 \bar{\zeta}[3,2] \\
& +\bar{\zeta}[6]-12 \bar{\zeta}[4,2]-3 \bar{\zeta}[4,1,1]+3 \bar{\zeta}[3,2,1] \stackrel{?}{=} 0 .
\end{aligned}
$$

An interesting point is that we obtain some relations of MZV's from the formulae above. Multiply $(1-q)^{6}$ and take the limit $q \rightarrow 1-$ in (15). Then the terms of weight less than 6 vanish and as a result we find

$$
\begin{array}{r}
-\zeta(6)-3 \zeta(4,2)+6 \zeta(3,3)=0 \\
\zeta(6)-12 \zeta(4,2)-3 \zeta(4,1,1)+3 \zeta(3,2,1)=0 .
\end{array}
$$

These relations for MZV's are correct [4].

The relations (15) are not derived from the cyclic sum formula and the Ohno relation because they are homogeneous with respect to weight. Unfortunately, it seems that the relations (15) are not also derived from our $q$-analogue of the Ohno-Zagier relations. By expanding the right hand side of (5) with the $q$-stuffle rule (6), choosing the part of weight 6 , and dividing both sides by $z-x y$, we obtain the coefficients as follows:

$$
\begin{aligned}
& x^{4}: 0=0, \\
& y^{4}: \bar{\zeta}[2,1,1,1,1]=\bar{\zeta}[5], \\
& x^{3} y: \bar{\zeta}[5,1]=\bar{\zeta}[6]-\bar{\zeta}[4,2]-\bar{\zeta}[3,3]-\bar{\zeta}[2,4], \\
& x y^{3}: \bar{\zeta}[3,1,1,1]=\bar{\zeta}[6]-\bar{\zeta}[4,2]-\bar{\zeta}[3,3]-\bar{\zeta}[2,4], \\
& x^{2} y^{2}: \bar{\zeta}[4,1,1]=\bar{\zeta}[6]+\bar{\zeta}[2,2,2]-\bar{\zeta}[2,4]-\bar{\zeta}[4,2]-2 \bar{\zeta}[3,3], \\
& x^{2} z: 0=0,
\end{aligned}
$$




$$
\begin{gathered}
\begin{array}{c}
y^{2} z: \bar{\zeta}[2,2,1,1]+\bar{\zeta}[2,1,2,1]+\bar{\zeta}[2,1,1,2] \\
=\bar{\zeta}[4,2]+\bar{\zeta}[3,3]+\bar{\zeta}[2,4] \\
x y z: \bar{\zeta}[3,1,2]+\bar{\zeta}[2,3,1]+\bar{\zeta}[3,2,1]+\bar{\zeta}[2,1,3] \\
\quad=-2 \bar{\zeta}[2,2,2]+\bar{\zeta}[2,4]+\bar{\zeta}[4,2]+2 \bar{\zeta}[3,3] \\
z^{2}: 0=0
\end{array}
\end{gathered}
$$

These are also homogeneous and do not lead to (15).

\section{Acknowledgment}

The authors wish to thank Kaneko Masanobu, Ohno Yasuo and David Bradley for many helpful informations and comments. The first author is supported by Grantin-Aid for Young Scientists (B) No. 17740026 and the second author is supported by Grant-in-Aid for Young Scientists (B) No. 17740089.

\section{References}

[1] Bradley, D. M.: 2005, 'Multiple q-zeta values'. J. Algebra 283(2), 752-798. Preprint: February 6, 2004, http://arxiv.org/abs/math.QA/0402093

[2] Gasper, G. and M. Rahman: 1990, Basic hypergeometric series, Vol. 35 of Encyclopedia of Mathematics and its Applications. Cambridge: Cambridge University Press. With a foreword by Richard Askey.

[3] Hoffman, M. E. and Y. Ohno: 2003, 'Relations of multiple zeta values and their algebraic expression'. J. Algebra 262(2), 332-347.

[4] Kaneko, M.: 2004. private communications.

[5] Ohno, Y.: 1999, 'A generalization of the duality and sum formulas on the multiple zeta values'. J. Number Theory 74(1), 39-43.

[6] Ohno, Y. and D. Zagier: 2001, 'Multiple zeta values of fixed weight, depth, and height'. Indag. Math. (N.S.) 12(4), 483-487.

[7] Terasoma, T.: 2002, 'Mixed Tate motives and multiple zeta values'. Invent. Math. 149(2), 339-369.

[8] Zagier, D.: 1994, 'Values of zeta functions and their applications'. In: First European Congress of Mathematics, Vol. II (Paris, 1992), Vol. 120 of Progr. Math. Basel: Birkhäuser, pp. 497-512.

[9] Zhao, J.: 2003, ' $q$-Multiple Zeta Functions and $q$-Multiple Polylogarithms'. preprint. May 23, 2003, http: //arxiv.org/abs/math.QA/0304448 